\title{
Short-Term Reliability Evaluation of Transmission System under Strong Wind and Rain
}

\author{
Qing Yang1, Xiaofu Xiong², Yanan Wei², Jian Wang2, Shijie Weng2 \\ ${ }^{1}$ Yunnan Power Grid Corporation, Kunming, China \\ ${ }^{2}$ State Key Laboratory of Power Transmission Equipment \& System Security and New Technology, \\ Chongqing University, Chongqing, China \\ Email: weiyanan1989@foxmail.com
}

Received February 2014

\begin{abstract}
The impact of strong wind and rain loads will adversely affect the reliability of the overhead lines, it's necessary to study changes in risk of transmission system and establish the reliability model of overhead lines through the strong wind and rain loads. In this article, the stochastic properties of overhead lines' strength and loads were used, according to principles of structural reliability, time-dependent failure probability model of overhead lines was established under the impact of strong wind and rain loads. Simulation of the IEEE-79 system demonstrates that failure probability model is effective. This simulation result also shows that the impact of strong wind and rain loads will seriously affect reliability indices of system loads, rain loads cannot be ignored under strong wind and rain loads.
\end{abstract}

\section{Keywords}

Strong Wind and Rain Loads; Transmission Line; Structural Reliability; Reliability Modeling

\section{Introduction}

The overhead line is susceptible to strong winds, heavy rain affected [1]-[3], for example, the violent typhoon bring on numerous damages in the electrical equipment of coastal areas, the impact of strong wind and rain loads will cause the accident of overhead lines and tower fracture [4] [5]. However, current reliability research of overhead lines rarely consider the impact of strong wind and rain loads, the current design criteria of overhead lines focuses on wind and ice loads [6]-[9] and ignores the impact of the rain loads, the impact of strong wind and rain loads will cause huge damage to tall building, especially overhead lines, those accidents [10] attract experts' attention of electricity and engineering widespread. References [11]-[14] study the effect of different rainfall intensity on the vertical drag coefficient of overhead lines in wind tunnel experiment, but they don't do the analysis of the mechanism. Reference [12] established a precise finite element model of overhead lines and studied rain-wind-induced dynamic response of transmission tower, but they didn't study the effect of wind and rain loads' impact to reliability of the overhead lines. 
In this article, the stochastic properties of overhead lines' strength and loads were used, according to principles of structural reliability, time-dependent failure probability model of transmission line was established under the impact of strong wind and rain loads, the effect of rain loads to failure probability of overhead lines is established. In simulation of the IEEE-79 system, the reliability indices of system loads are calculated considering no environmental loads, only wind loads, wind and rain loads.

\section{Strong Wind and Rain Loads of Overhead Lines}

\subsection{Wind Loads}

According to theGB50545-2010 of code for design of $110 \mathrm{kV}$ - $750 \mathrm{kV}$ overhead transmission line [9], the wind loads of overhead lines:

$$
F_{w}(t)=\alpha \cdot \frac{V^{2}(t)}{1600} \cdot \mu_{z} \mu_{s c} \beta_{c} d L_{v} B \sin ^{2} \theta
$$

where $\alpha$ is coefficient of uneven wind pressure and is related to the wind speed; $V(t)$ is standard wind speed; $\mu_{Z}$ is coefficient of wind pressure changes with altitude and is related to the surface roughness and altitude of overhead lines; $\mu_{s c}$ is shape coefficient of overhead lines, it is equal to 1.2 if the line diameter is less than $17 \mathrm{~mm}$ or ice depositson lines, it is equal to 1.1 if the line diameter is greater than or equal to $17 \mathrm{~mm}$; $\beta_{\mathrm{c}}$ is wind vibration coefficient, only the overhead lines of $500 \mathrm{kV}$ and $750 \mathrm{kV}$ are equal to 1.25 , the others are equal to $1 ; d$ is the diameter of the line; $L_{v}$ is Horizontal Span; $B$ is the intensification coefficient of wind loads when ice depositson lines, it is equal to $1.1,1.2,1$ if the ice thickness is $5 \mathrm{~mm}, 10 \mathrm{~mm}, 0 \mathrm{~mm}$; $\theta$ is the angle between the wind direction and the line.

\subsection{Rain Loads}

The rainfall patterns are divided into seven levels according to intensity of rainfall as shown in Table 1 [13]:

Rain load calculation equation is as follows [12]:

$$
F_{r}(t)=\frac{2}{9} \pi d^{3} n b V_{s}^{2}
$$

where $d$ is raindrop diameter, $n$ is the number of raindrops per unit volume, $b$ is the area of scoured by rain, $V_{s}$ is the final velocity of raindrops.

Observational data suggest that $d$ is usually as follows:

$$
n(d)=n_{0} \exp (-\Lambda d)
$$

where $n_{0}=8 \times 10^{3}\left(1 / \mathrm{m}^{3} \cdot \mathrm{mm}\right) ; \quad \Lambda$ is slope factor, $\Lambda=4.1 I^{-0.21}, \quad I$ is intensity of rainfall $(\mathrm{mm} / \mathrm{h})$.

\subsection{Combinations of Loads}

Loads are divided into three parts: wind loads $F_{W}(t)$, rain loads $F_{r}(t)$ and fixed loads $G$. Fixed load, also known as permanent loads, is a Gaussian distribution, mainly components weight; wind and rain loads are variable load and they are the function of time-dependent. Due to the feature of probability distribution function, if the random variable $x$ is a Gaussian distribution and mean value of $x$ and standard deviation are $\mu$ and $\delta$, then random variable $(x+a)$ is also Gaussian distribution and mean value and standard deviation are $\mu+a$ and $\delta$, where $a$ is a constant.

Consider the most serious cases, namely the wind and rain loads and the permanent loads are in the same direction:

Table 1. The class of rain.

\begin{tabular}{ccccccc}
\hline \multirow{2}{*}{ Level } & Moderate rain & Heavy rain & Rainstorm & \multicolumn{3}{c}{ Downpour } \\
\cline { 3 - 7 } & & & & Weak & Middle & Strong \\
\hline Intensity of rainfall $(\mathrm{mm} / \mathrm{h})$ & 8 & 16 & 32 & 64 & 100 & 200 \\
\hline
\end{tabular}




$$
S(t)=G+Q(t)=G+F_{w}(t)+F_{r}(t)
$$

where $S(t)$ is the total loads of overhead lines, $G$ is permanent loads, $Q(t)$ is environmental loads, it is the sum of wind loads $F_{w}(t)$ and rain loads $F_{r}(t)$, due to the IEC60826, the permanent loads is gaussian distribution, its mean value and standard deviation are $\mu_{G}$ and $\delta_{G}$, so the total loads is also Gaussian at time t, the mean value And standard deviation are $\mu_{G}+F_{w}(t)+F_{r}(t)$ and $\delta_{G}$.

\section{Reliability Modeling}

According to the structure reliability theory, an overhead lines' analytical model of reliability analysis is established under the impact of strong wind and rain loads. The structure state function of the transmission line is expressed as a random process:

$$
Z(t)=g(R, S(t))=R-S(t)
$$

where $R$ is the design strength of the overhead lines, according to the IEC, $R$ is a Gaussian random variable; $S(t)$ is the total loads of overhead lines. When the loads of overhead lines are less than the design strength, the lines running normally; conversely, the line will fail.

\subsection{Failure Probability of Overhead Lines under the Impact of the Strong Wind and Rain Loads}

The relationship between the strength probability density distribution and permanent loads probability density distribution is as shown in Figure 1.

It is the relationship between the strength probability density distribution and permanent loads probability density distribution taking no account of the environmental loads as shown in Figure 1(a), the line is reliable because of the design strength of the line is greater than the permanent load; the relationship between the strength probability density distribution and permanent loads probability density distribution considering the environmental loads is shown in Figure 1(b), there are overlap which is called as interference region between the curve, the interference region have to emerge due to the increase of environmental loads which is result in increase of loads of lines, it has potential possibility of lines fault because of this interference region.

The probability of failure of overhead lines is equal to the probability which the strength is less than the loads of lines:

where $P(f)$ is probability of failure of overhead lines,

$$
P_{f}(t)=P(Z(t)<0)=P(R-S(t)<0)
$$

Strength $R$ and loads $S$ is independent, the joint probability density function is

$$
f_{Z}=f_{R}(r) \cdot f_{s}(s)
$$

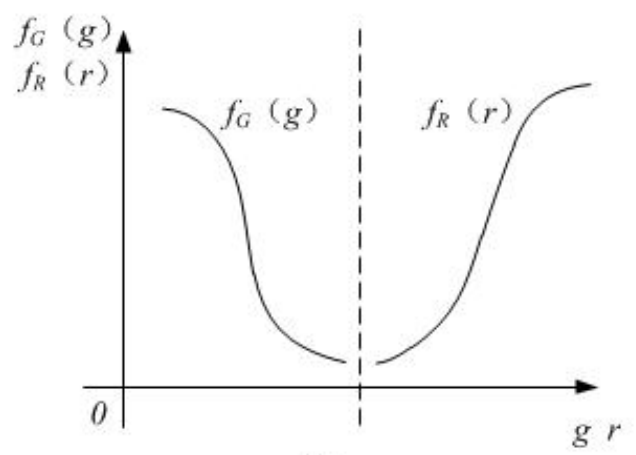

(a)

$f_{G}(g)$ permanent loads probability density distribution $f_{R}(r)$ strength probability density distribution

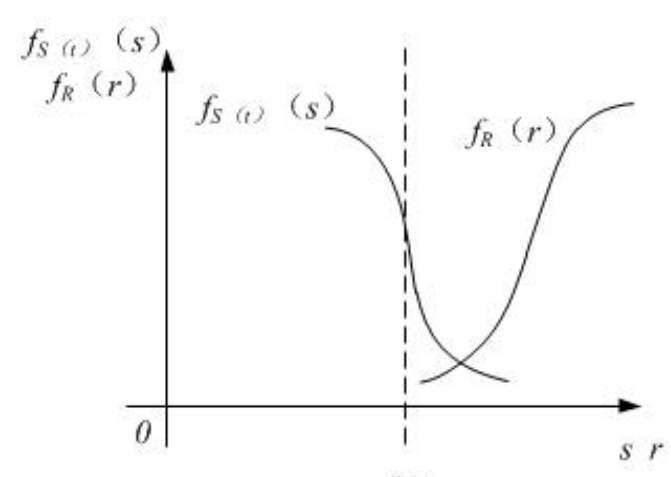

(b)

$f_{S}(t)(s)$ loads probability density distribution at time t $f_{R}(r)$ strength probability density distribution

Figure 1. The interferogram of strength and loads probability density curve. 
where $f_{R}(r)$ and $f_{s}(s)$ are the probability density function of strength and loads, $f_{z}$ is the joint probability density function, the Equation (6) can be represented by the Equation (8):

$$
P_{f}(t)=P(R<S(t))=P(r<s)=\iint f_{R}(r) \cdot f_{S}(s) d r=\int_{0}^{+\infty} f_{R}(r) F_{S}(r) d r
$$

According to the IEC 60826, the strength of lines is gaussian distribution, then $f_{z}$ can be represented by the following equation:

$$
f_{Z}=\frac{1}{\sqrt{2 \pi} \sigma_{z}} \exp \left(-\frac{\left(z-\mu_{z}\right)^{2}}{2 \sigma_{Z}^{2}}\right)
$$

where $\mu_{Z}=\mu_{R}-\mu_{S}, \sigma_{Z}=\sigma_{R}-\sigma_{S}, \mu_{R}, \mu_{S}$ are probability density function mean value of strength and loads, $\sigma_{R}, \sigma_{S}$ are the probability density function standard deviation of strength and loads, $\mu_{Z}, \sigma_{Z}$ are the mean value and standard deviation of joint probability density function

Equation (8) can be expressed as:

$$
P_{f}(t)=P(Z(t)<0)=\int_{-\infty}^{0} \frac{1}{\sqrt{2 \pi} \sigma_{Z}} \exp \left(-\frac{\left(z-\mu_{Z}\right)^{2}}{2 \sigma_{Z}^{2}}\right) d z
$$

$A=(z-\mu) / \sigma_{Z}$, Equation (10) can be expressed as Standard normal distribution:

$$
P_{f}(t)=P(Z(t)<0)=\int_{-\infty}^{-\frac{\mu_{Z}}{\sigma_{Z}}} \frac{1}{\sqrt{2 \pi}} \exp \left(-\frac{\alpha^{2}}{2}\right) d \alpha
$$

the probability of failure of overhead lines is

$$
P_{f}(t)=\phi\left(-\frac{\mu_{Z}}{\sigma_{Z}}\right)
$$

For an entire overhead line, it can be divided into $n$ lines as shown in Figure 2, so its probability of failure is equal to series probability of failure of $n$ lines.

In Figure 2, where $p_{f(\mathrm{i})}$ is the probability of failure of the $i$-th line, $p_{f(\mathrm{n})}$ is the probability of failure of the $n$-th line, $P_{f(\mathrm{n})}$ is equivalent probability of failure of $\mathrm{n}$ series lines, according to the characteristic of series network, the probability of failure of $\mathrm{n}$ series lines is

$$
P_{f(n)}(t)=P_{f(n-1)}(t)+p_{f(n)}(t)-P_{f(n-1)}(t) \cdot p_{f(n)}(t), n \geq 2
$$

where $P_{f(n-1)}(\mathrm{t})$ is equivalent probability of failure of $n-1$ series lines at time $\mathrm{t}, p_{f(n)}(\mathrm{t})$ is the probability of failure of the $n$-th line at time $t$.

\subsection{Short-Term Reliability Assessment of System under the Impact of Strong Wind and Rain}

Short-term system reliability assessment process of generation and transmission System under the impact of strong wind and rain loads is shown in the follow:

1) Getting short-term load forecasting data and generator combinations;

2) Getting weather forecast information from weather bureau, it includes wind speed, intensity of rainfall and regions;

3) Using time-dependent failure probability model of overhead lines to solve the time-dependent failure probability of lines in the region of strong wind and rain;

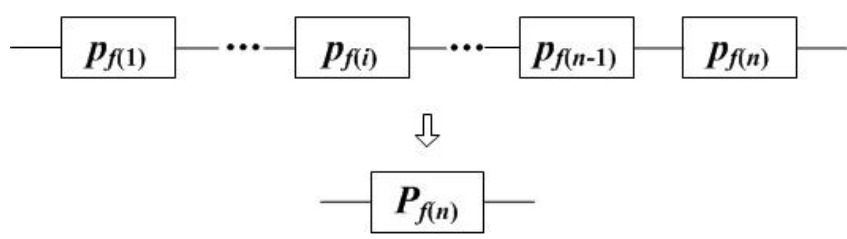

Figure 2. Series equivalence failure probability of Overhead lines. 
4) Using state sampling approach to simulate;

5) Analyzing the state of system, using the optimal cutting load model to calculate removal of the load;

6) Calculating reliability indices of system loads.

In this paper, using loss of load probability (LOLP), expected demand not supplied (EDNS) and expected energy not supplied (EENS) to express reliability indices.

$L O L P$ : The probability of the system can't satisfy the load demand within a certain period of time:

$$
L O L P=\sum_{s \in F} \frac{m(s)}{M}
$$

where $M$ is the total number of system status, $m(s)$ is the number of system state $s$ in the sampling.

EDNS: System expected demand not supplied due to insufficient generation capacity or grid constraints within a certain period of time:

$$
E D N S=\sum_{s \in F} \frac{m(s)}{M} \times C(s)
$$

where $C(s)$ is removal of load at system state $s$.

EENS: System expected energy not supplied due to insufficient generation capacity or grid constraints within a certain period of time:

$$
E E N S=\sum_{s \in F} \frac{m(s)}{M} \times C(s) \times t
$$

where $t$ is a certain period of time.

\section{Example}

\subsection{Example Introduction}

In this paper, we use Simulation of the IEEE-79 system as example. This system contains two voltage levels: $230 \mathrm{kV}$ and $138 \mathrm{kV}$, it is divided into two regions: region 1 and region 2 as shown in Figure 3. Using the line LGJ-300/40 (Diameter $23.94 \mathrm{~mm}$, CUTS $T_{m}=92.22 \mathrm{kN}$, Security Coefficient $K=2.5$, Unit Weight $Q=1133$

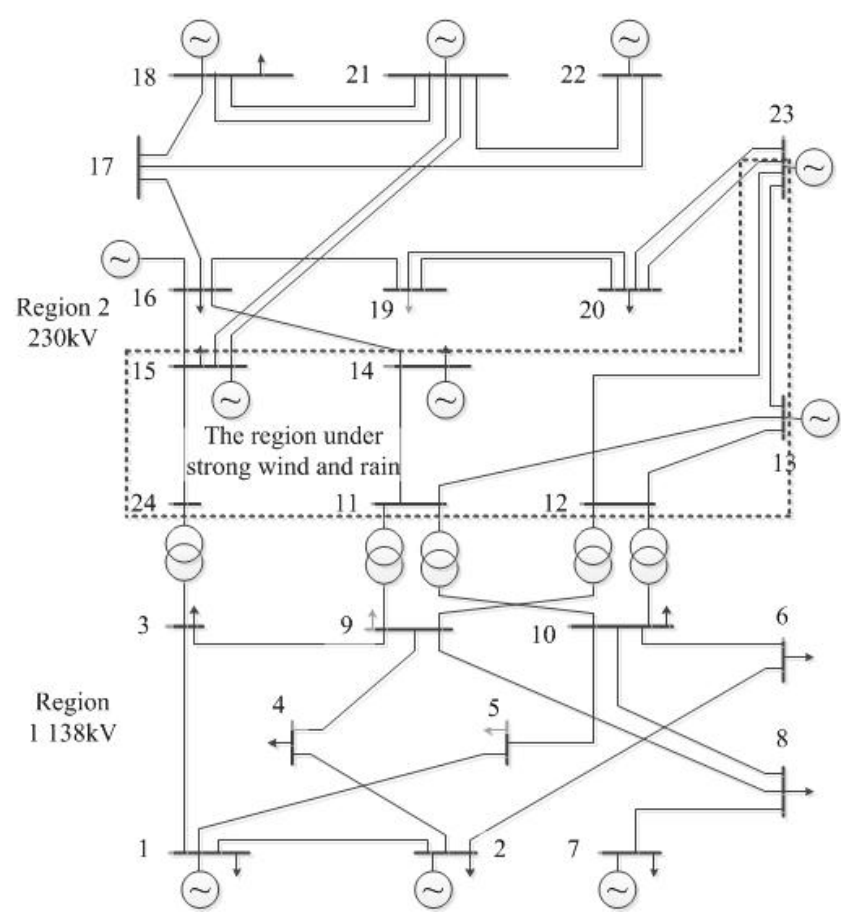

Figure 3. Electric diagram of IEEE RTS-79. 
$\mathrm{kg} / \mathrm{km}$ ) as example, Design wind speed is $27 \mathrm{~m} / \mathrm{s}$, Horizontal Span $l_{h}=350 \mathrm{~m}-820 \mathrm{~m}$, Vertical Span $l_{v}=450 \mathrm{~m}$ $1200 \mathrm{~m}$, according to the experience, the vertical span is equal to 1.25 to 1.7 times of horizontal span (in this paper, taking 1.5 times), the mean value coefficient of permanent loads is 1.06 , the coefficient of variation of permanent loads is 0.07 , the mean value coefficient of strength is 1.0917, the coefficient of variation of strength is 0.0915 .

Line 11 - 13 (53 km), line 11 - 14 (47 km), line 12 - 13 (53 km), 12 - 23 (108 km), line 13 - 23 (97 km), line 15 - 24 (58 km) are in the region under strong wind and rain.

\subsection{Failure Probability of Overhead Lines under the Impact of the Strong Wind and Rain Loads}

Setting a standard structure model of "three towers two cross", the two spans are $l_{1}=400 \mathrm{~m}$ and $l_{2}=600 \mathrm{~m}$, then the horizontal span and vertical span are

$$
l_{h}=\frac{l_{1}+l_{2}}{2}=500 \mathrm{~m}, \quad l_{v}=1.5 l_{h}=750 \mathrm{~m}
$$

The weight of overhead line is

$$
G_{d}=10 Q \cdot l_{v}=10 \times 1133 \times 0.75=8.5 \mathrm{kN}
$$

the mean value and standard deviation of the load are

$$
\begin{aligned}
& \mu_{S}=F_{w}(t)+F_{r}(t)+9.01 \\
& \sigma_{s}=0.63
\end{aligned}
$$

The broken tension of line is equal to the percentage of maximum tension. In this paper, taking it as 0.5 , the broken tension is

$$
T_{d}=0.6 T_{m}=0.5 T_{p} / K=0.6 \times 92.22 / 2.5=22.13 \mathrm{kN}
$$

The mean value and standard deviation of the line strength are

$$
\mu_{R}=24.16, \sigma_{R}=2.21
$$

Using Equation (12) to calculate the failure probability of lines under strong wind and rain in one span, the failure probability of lines for different levels wind speed and rain is shown in Figure 4.

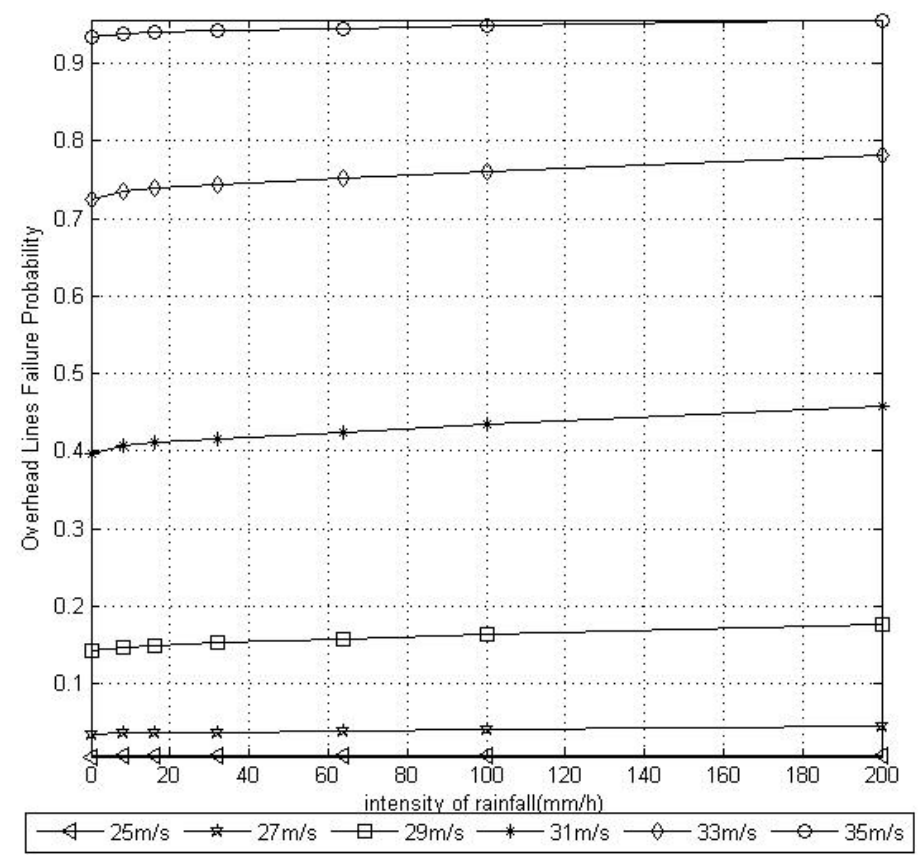

Figure 4. Overhead lines failure under strong wind and rain loads. 
We can get the following conclusions from the above Figure 4: 1) the failure probability of lines will increase with wind loads increase; 2) Rain loads have influence upon the failure probability of lines, and heavy rainfall have greater influence on the failure probability of lines when the wind speed is high.

Failure probability of overhead lines under different levels of rainfall is shown in Table 2 (the wind speed is design speed). As can be seen from the table, the failure probability of overhead lines increases by $30.03 \%$ when consider the influence of strong rainfall. So the influence of strong rainfall can't be ignored when calculating the failure probability of overhead lines in engineering applications.

\subsection{Short-Term Reliability Assessment of System under the Impact of Strong Wind and Rain}

In order to analyze how the impact of strong wind and rain loads influence the short-term reliability indices of system, we do not consider the changes of and generator combinations. In simulation of the IEEE-79 system, the reliability indices of system loads are calculated considering No environmental loads, only wind loads, wind and rain loads.

The wind speed and intensity of rainfall cure of somewhere during 10 hours are shown in Figure 5.

The cure of system EENS in the above three cases is shown in the Figure 6.

A significant reduction can be seen from Figure 6 when considering the environmental loads; the system EENS considering wind and rain loads is $31.72 \%$ higher than the EENS without considering rain loads.

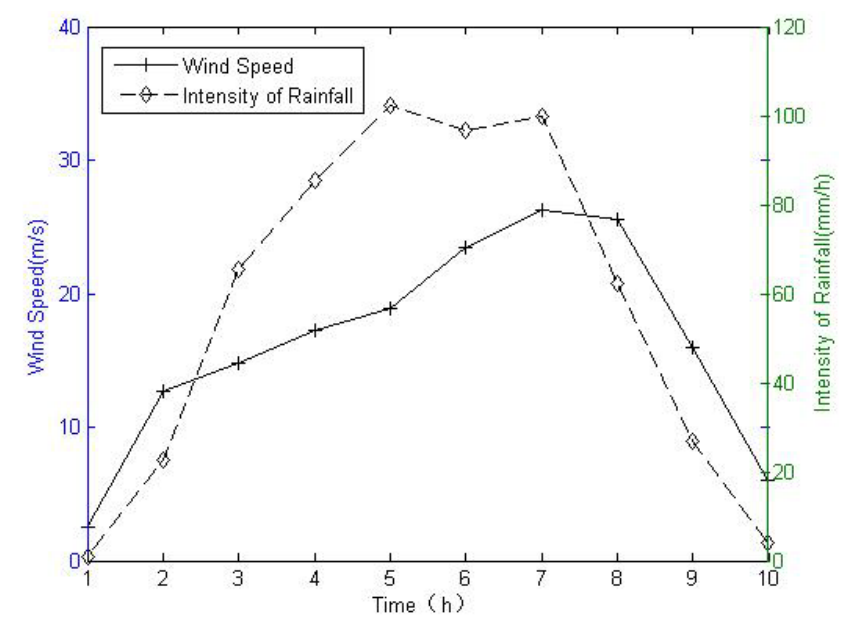

Figure 5. Typhoon winds and rainfall within 10 hours.

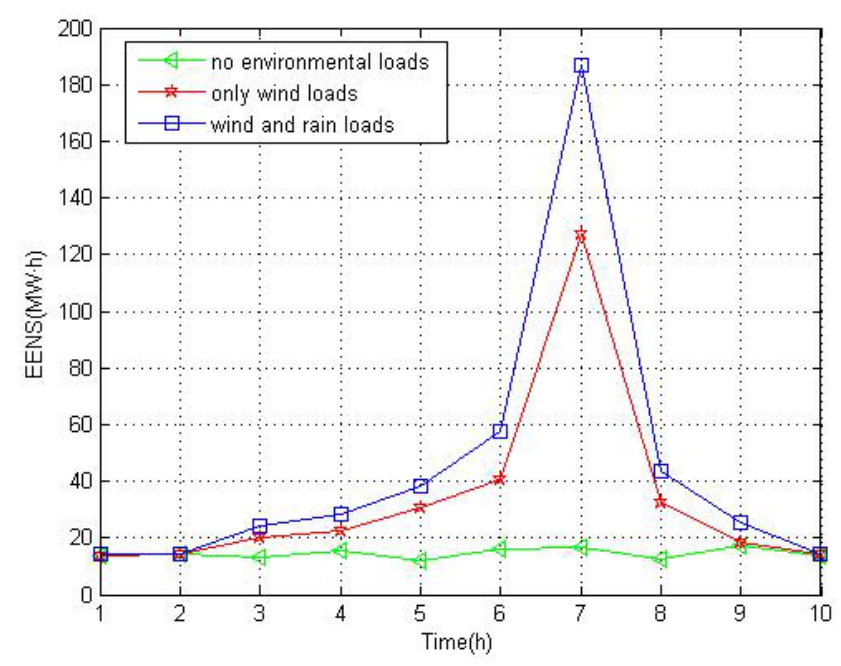

Figure 6. The curve of system EENS over time. 
Table 2. Failure probability of overhead lines under different levels of rainfall.

\begin{tabular}{ccccccc}
\hline \multirow{2}{*}{ Load } & \multirow{2}{*}{ Wind } & Wind and Rain (Heavy rain) & Wind and Rain (Rainstorm) & \multicolumn{3}{c}{ Wind and Rain (Downpour) } \\
\cline { 6 - 8 } & & 0.0355 & Weak & Middle & Strong \\
\hline Failure probability & 0.0333 & 6.61 & 0.0364 & 0.0377 & 0.0394 & 0.0433 \\
Increment/\% & - & 9.31 & 13.21 & 18.32 & 30.03 \\
\hline
\end{tabular}

\section{Conclusions}

In this paper, analyzing the influence which impact of strong wind and rain loads produced to the reliability of the overhead lines, establishing the failure probability wind and rain loads, the conclusions are shown as follow:

1) The failure probability model of overhead lines under the strong wind and rain loads are different from the normal state;

2) The rain loads can't be ignored when calculating the failure probability of overhead lines under the strong wind and rain loads;

3) Using the weather forecast to assess system reliability, it is contributed to prepare for proper risk control measures and improve the ability of withstanding severe weather for power system.

\section{References}

[1] Liu, Y. and Zhou, J.Q. (2003) Incorporating Weather Effect in Bulk Power System Reliability Evaluation. Electric Power Automation Equipment, 23, 60-62.

[2] Li, Y.H., Han, J.Y. and Wang, T. (2009) Ice Monitoring System of Overhead Electrical Power Lines. Electric Power Automation Equipment, 29, 112-115.

[3] Li, P. and Ren, Z. (2002) Guangzhou Regional Load Analysis and Short-Term Forecasting Model Design. Electric Power Automation Equipment, 22, 50-53.

[4] Tang, S.Q., Zhang, M., Jian, S., Wu, X.C., Jiang, K. and Shu, S.Y. (2006) Review of blackout in Hainan on September 26th-Cause and Recommendations. Automation of Electric Power Systems, 30, 1-7, 16.

[5] Pang, H., Li, B.F., Yu, Y.E, Li, X., Wu, X.C., Zhao, Y., Yu, Q.Y. and Li, P. (2007) Study on Operating Modes of Hainan Power Grid during Typhoon Periods. Power System Technology, 31, 46-50.

[6] Sun, Y., Wang, X.L., Wang, J.X., and Xie, S.Y. (2011) Wind and Ice Loading Risk Model and Fuzzy Forecast for Overhead Transmission Lines. Proceedings of the CSEE, 31, 21-28.

[7] Wang, J.X., Zhang, Y., Wu, S. and Sun, Y. (2011) Influence of Large-Scale Ice Disaster on Transmission System Reliability. Proceedings of the CSEE, 28, 49-56.

[8] (2003) IEC 60826 Design Criteria of Overhead Transmission Lines.

[9] (2010) GB50545-2010 of Code for Design of $110 \mathrm{kV}$ - $750 \mathrm{kV}$ Overhead Transmission Line.

[10] Xie, Q., Zhang, Y. and Li, J. (2006) Investigation on Tower Collapses of 500 kV Renshang 5237 Transmission Line Caused by Downburst. Power System Technology, 30, 59-53.

[11] Eguehi, Y., Kikuehi, N. and Kawabata, K. (2002) Drag Reduction Mechanism and Aerodynamic Characteristics of a Newly Developed Overhead Electric Wire. Journal of Wind Engineering and Industrial Aerodynamics, 90, 293-304. http://dx.doi.org/10.1016/S0167-6105(01)00201-X

[12] Bai, H.F. and Li, H.N. (2009) Dynamic Response of Overhead Transmission Lines to Oscillation Caused by Wind or Rainfall Loads. Power System Technology, 33, 36-40.

[13] Sheng, P.X., Mao, J.T. and Li, J.G. (2003) Atmospheric Physics. Peking University Press, Beijing.

[14] Kikuehi, N. and Matsuzaki, Y. (2003) Aerodynamic Drag of New-Design Electric Power Wire in a Heavy Rainfall and Wind. Journal of Wind Engineering \& Industrial Aerodynamics, 91, 41-45. http://dx.doi.org/10.1016/S0167-6105(02)00334-3 